\title{
Broadband Terahertz Time-Domain Spectroscopy and Low-Frequency Raman Scattering of Propylene Glycol
}

\author{
Shota Koda ${ }^{1, a^{*}}$, Tatsuya Mori ${ }^{1, b}$, Seiji Kojima ${ }^{1, c}$ \\ ${ }^{1}$ Graduate School of Pure and Applied Sciences, University of Tsukuba, Tsukuba, Ibaraki 305-8573, \\ Japan

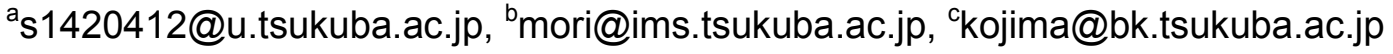

Keywords: propylene glycol, terahertz time-domain spectroscopy, low-frequency Raman scattering

\begin{abstract}
Two different THz-TDS systems were used for the accurate study of broadband terahertz dynamics between 0.1 and $5.0 \mathrm{THz}$. These systems enabled to observe the whole spectral structure of the broad peak at $1.5 \mathrm{THz}$ in propylene glycol. At high-frequency side of the broad peak in the dielectric spectrum, the small shoulder peak at $3.6 \mathrm{THz}$ was also observed. It is assigned to the inter-molecular vibration, which is supported by the precedent report and the Gaussian calculation. In contrast, the low-frequency side of the broad peak includes the contribution of the relaxation processes which exist in the $\mathrm{MHz}$ and $\mathrm{GHz}$ region. Furthermore, we compared the dielectric spectrum with the Raman spectrum and concluded that the mode at $3.6 \mathrm{THz}$ is IR active while Raman inactive.
\end{abstract}

\section{Introduction}

The $\mathrm{THz}$ dynamics have been intently investigated for various kinds of materials such as polymers [1], pharmaceuticals [2], ferroelectrics [3], and so on. Furthermore, there has been the significant improvement of the methods for measuring not only the crystalline state, but also the amorphous and liquid states of materials. Most of the glassy and liquid states materials have a broad peak in $\mathrm{THz}$ region, and thus it should be discussed with a broadband $\mathrm{THz}$ spectrum; however, unfortunately for the liquid states, the investigation in the $\mathrm{THz}$ region reached at most $2.5 \mathrm{THz}$ even in the recent studies due to the high absorption of the liquids [4,5]. Therefore, those of the higher frequency $\mathrm{THz}$ dynamics remain unsolved. Recently, new THz-TDS measurement technique has been established using high-speed asynchronous optical sampling (AOS) technique, and enables to measure the high frequency region [6].

Infrared and Raman scattering spectroscopy observe the molecular dynamics through the change of dipole moment and polarizability, respectively. Dielectric loss $\varepsilon$ " and the imaginary part of Raman susceptibility $\chi$ " have been often compared and discussed [7]. For molecules and crystals, the selection rule holds for infrared (IR) active and Raman active modes according their point groups. In contrast for liquids, there is little difference in the frequency shift of a vibrational state, while dipolar aprotic liquids were reported that the relaxation mode of $\varepsilon$ " deviate from that of $\chi$ " [7]. It is significant to investigate a same sample by using both far-IR and low-frequency Raman spectroscopies.

Propylene glycol (PG) is a typical intermediate glass-forming molecular liquid and commonly used for studies of the glass transition which is related to the dynamics of super-cooled liquids [8,9]. Furthermore, the PG esters with no hydroxyl group, which are the modification of PG, have been used for the pharmaceutical application recently [10]. Therefore, the investigation of the relaxation process in the unsolved $\mathrm{THz}$ region for a simple glass-forming PG is important.

This work is aimed at the investigation of the broadband $\mathrm{THz}$ dynamics using two types of terahertz time-domain spectroscopy (THz-TDS) systems, and the accurate analysis of $\mathrm{THz}$ dynamics with the computational calculation using the Gaussian. Furthermore, the combination the results of THz-TDS with those of Raman scattering enable us to discuss the discrepancy between $\varepsilon$ " and $\chi ”$. 


\section{Experimental \& Calculation Methods}

In the analysis of THz-TDS spectra, the observed spectra of two THz-TDS systems are combined to cover a broad $\mathrm{THz}$ region $[1,11]$. The low frequency sides of $\mathrm{THz}$ transmission spectra were measured in the frequency range from 0.1 to $3.0 \mathrm{THz}$ using a standard THz-TDS equipment (RT-10000, Tochigi Nikon Co.) with low temperature-grown GaAs photoconductive antennas for both the emitter and detector. The photoconductive antennas were triggered by a mode-locked Ti:sapphire pulsed laser with a wave-length of $780 \mathrm{~nm}$, a pulse width of less than $100 \mathrm{fs}$, and a repetition rate of $80 \mathrm{MHz}[1,11]$. The high frequency sides of $\mathrm{THz}$ transmission spectra were measured in the frequency range from 1.0 to $5.0 \mathrm{THz}$ using an recently developed THz-TDS equipment (TAS7500SU, ADVANTEST Co.) with a Cherenkov type THz generator and the high speed AOS technique $[1,11]$. Since the difference in the spectra observed by these systems was at most $5 \%$, we smoothly connected the absolute value of the complex dielectric constants of the samples by multiplying a factor in the data analysis.

We performed three kinds of measurements, which are background, liquid cell (window) only, and liquid sample inserted in the cell as shown in Fig. 1. The Z-cut quartz was used for the window in the measurement of low frequency sides (RT-10000) and high density polyethylene was used for the window in the measurement of high frequency sides (TAS7500SU). The thicknesses of the window and the sample are $5.0 \mathrm{~mm}$ and $0.296 \mathrm{~mm}$, respectively in the measurement of RT-10000, and $3.0 \mathrm{~mm}$ and $0.200 \mathrm{~mm}$, respectively in the measurement of TAS7500SU. Figure 2(a) shows the measured time-domain $\mathrm{THz} E$-field waveforms transmitted through the air (background), window, and PG observed in the THz-TDS system of RT-10000, and Fig. 2(b) shows the frequency-domain power spectra. Figure 3 shows those observed in the THz-TDS system of TAS7500SU. The absorption dip observed at $3.8 \mathrm{THz}$ in Fig. 2(b) is due to the $\mathrm{E}_{1}$ mode which is the deformational vibration of silicon atoms in the quartz [12,13]. Figure 3(b) also showed the absorption dip at 2.2 $\mathrm{THz}$, and it is originated from the $\mathrm{B}_{1 \mathrm{u}}$ lattice mode in high density polyethylene $[14,15]$. From the comparison between the frequency-domain power spectra shown in Fig. 2(b) and Fig. 3(b), it can be clearly seen that the spectra measured by RT-10000 supports the low frequency side of those by using TAS7500SU. To calculate the frequency dependent complex dielectric constant $\varepsilon=\varepsilon$ ' $+i \varepsilon$ " from the measured time-domain waveforms, we have used following equation,

$$
\frac{E_{S}(v)}{E_{W}(v)}=t_{W S} t_{S W} \frac{\exp \left\{i\left(n_{S}-1\right) \omega d_{S} / c\right\}}{1-r_{S W}^{2} \exp \left(i 2 n_{S} \omega d_{S} / c\right)},
$$

where $E_{S}(v)$ and $E_{W}(v)$ are the amplitude spectra of $\mathrm{THz}$ pulse transmitted the sample and the window, respectively. $c$ and $d_{S}$ are the light velocity and a thickness of sample, respectively. $t_{i j}=$ $2 n_{i} /\left(n_{i}+n_{j}\right)$ and $r_{i j}=\left(n_{i}-n_{j}\right) /\left(n_{i}+n_{j}\right)$ are the complex Fresnel's transmission and reflection coefficients at the interface between regions $i$ and $j$, respectively. The subscripts $i$ and $j$ stand for $W, S$, representing the window and sample, respectively. $n_{i}$ is the complex refractive index of region $i$. Then, the complex dielectric constant is obtained from the relation of $\varepsilon=n^{2}$.

Depolarized $V H$ Raman scattering spectra were measured in the frequency range from 0.3 to 5.0 $\mathrm{THz}$ in the backward geometry with a scattering angle $\theta=180^{\circ}$, using a single frequency green-YAG laser with wavelength $532 \mathrm{~nm}$. The observed Raman intensity $\operatorname{spectra} I_{\text {raw }}(v)$ were converted to the imaginary part of the Raman susceptibility $\chi$ ” $(v)$ by the following equation,

$$
\chi^{\prime \prime}(v)=\frac{I_{r a w}(v)}{n_{B}(v)+1},
$$

where $n_{B}(v)=\left(\exp \left(h v / k_{B} T\right)\right)^{-1}$ is the Bose-Einstein distribution function. The sample of PG was purchased from Sigma-Aldrich, and studied without further purification, and geometry optimizations and the frequency calculations of PG was performed by density functional theory (DFT) using the Gaussian09 B3LYP/6-31G + $(d, p)$ level of the theory $[16,17]$. 
(a)

Background (b)

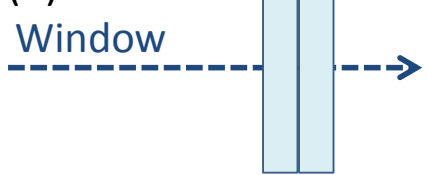

(c)

Sample

Figure 1. (a) The schematic diagrams of three kinds of $\mathrm{THz}$ transmittance measurements which consists of the transmittance of (a) air (background), (b) window (high density polyethylene or quartz), and (c) sample.
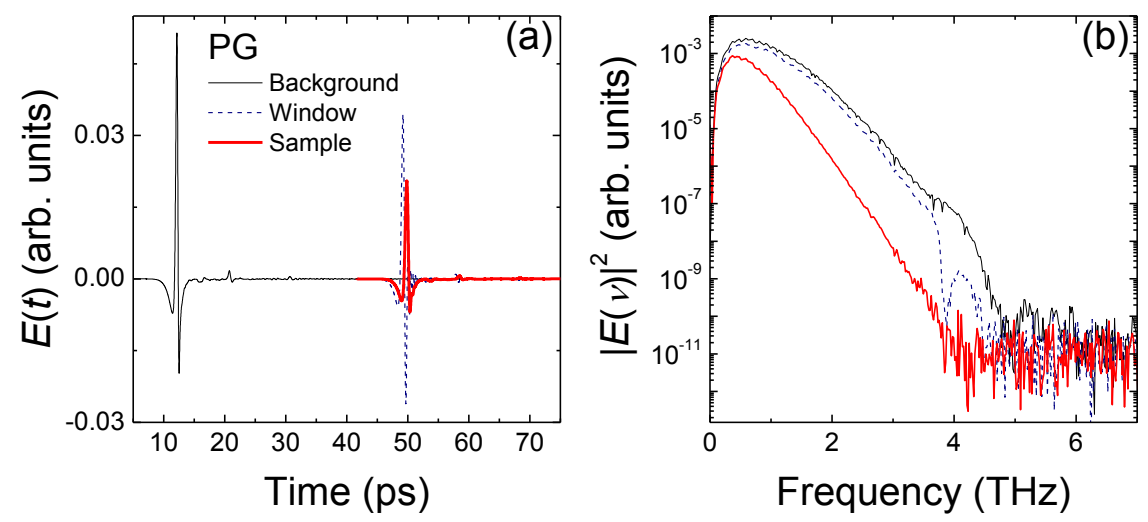

Figure 2. (a) Time-domain waveforms and (b) frequency-domain power spectra of background (black solid lines), window (blue dots lines), and PG (red solid lines) measured by RT-10000 (Tochigi Nikon corp.) at room temperature.
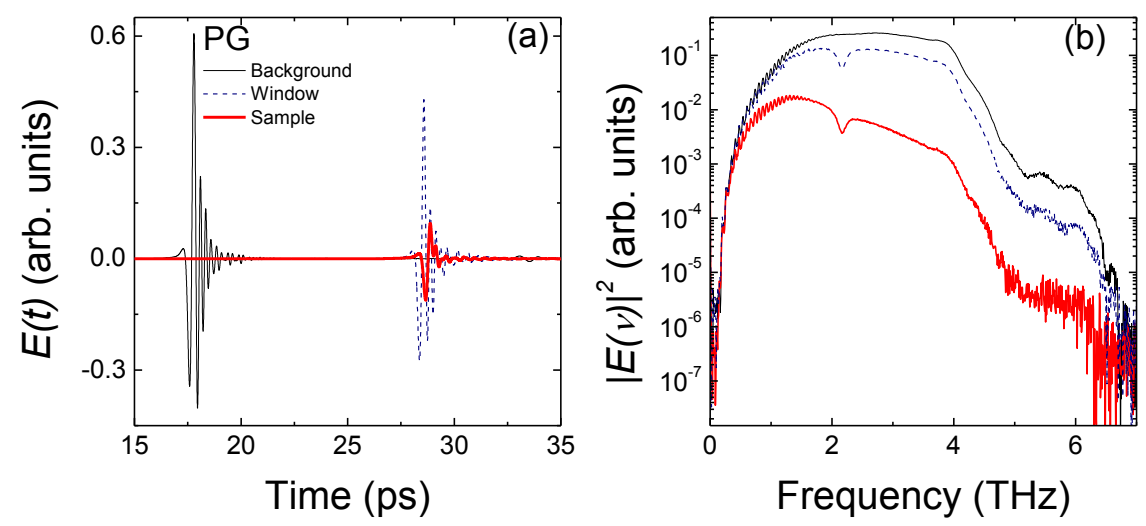

Figure 3. (a) Time-domain waveforms and (b) frequency-domain power spectra of background (black solid lines), window (blue dots lines), and PG (red solid lines) measured by TAS7500SU (ADVANTEST corp.) at room temperature.

\section{Results \& Discussion}

Figure 4 shows the complex dielectric constants and the $\chi$ ” in the broad THz region of PG. The combination of two THz-TDS systems enable to observe the broadband dielectric constants spectra and the whole structure of the broad peak at $1.5 \mathrm{THz}$ for $\varepsilon$ " in PG. The dielectric spectrum of PG consists of the broad peak at $1.5 \mathrm{THz}$ and the small shoulder at $3.6 \mathrm{THz}$, while only one peak at 1.5 $\mathrm{THz}$ seems to be observed in the $\chi$ " spectrum. The peaks at $1.5 \mathrm{THz}$ of alcohols have been discussed for some kinds of vibrational modes. Yomogida et al. observed the peak at $1.5 \mathrm{THz}$ of pentanol isomers and suggested to be the vibrational mode which is originated by the location of the 
$\mathrm{OH}$ groups and the carbon chain structure $[18,19]$. They fitted the peak at $1.5 \mathrm{THz}$ by using the damped harmonic oscillator (DHO) model, which is commonly used for fitting a vibrational mode.

The shoulder at $3.6 \mathrm{THz}$ observed in the dielectric spectrum of PG has never been reported so far. The Gaussian calculation for PG monomer shows no vibrational mode below $4.5 \mathrm{THz}$ as shown in Fig. 5. The precedent researches of glycerol reported two kinds of vibrational modes at around 3.0 $\mathrm{THz}$ for cyclic glycerol trimers. One is the collective motions of the $\mathrm{OH}$ groups and the other is the translational motions of the $\mathrm{CH}_{2}$ and $\mathrm{CH}$ groups [20,21]. These experimental and calculation results suggest that the observed small shoulder at $3.6 \mathrm{THz}$ can be attributed to not intra- but inter-molecular vibrations.

The low frequency side of $\varepsilon$ " suggests a contribution from the $\mathrm{GHz}$ region. The precedent work of the dielectric measurement of PG reported the $\alpha$ - and fast processes exist in the $\mathrm{MHz}-\mathrm{GHz}$ region, and the high frequency sides of the processes remain in the $\mathrm{THz}$ region at room temperature [8]. The comparison between the $\varepsilon$ " and $\chi$ " doesn't seem to show the relaxation process in the low-frequency region of PG. We have reported the wide frequency range from $\mathrm{GHz}$ to $\mathrm{THz}$ for $\mathrm{PG}$ of $\chi$ " using Raman scattering and Brillouin scattering [22]. This result showed the relaxation processes of the $\alpha$ - and fast processes; however, the relaxation strength of these relaxation process of $\chi$ " is much smaller than those of $\varepsilon$ " measured by Kohler et al [8]. The similar results were reported by Fukasawa et al. [7], and they suggested that the relaxation arising from the long range hydroxyl bond mediated dipole-dipole interactions gives the main contribution to the dielectric spectrum, and this process is Raman inactive.

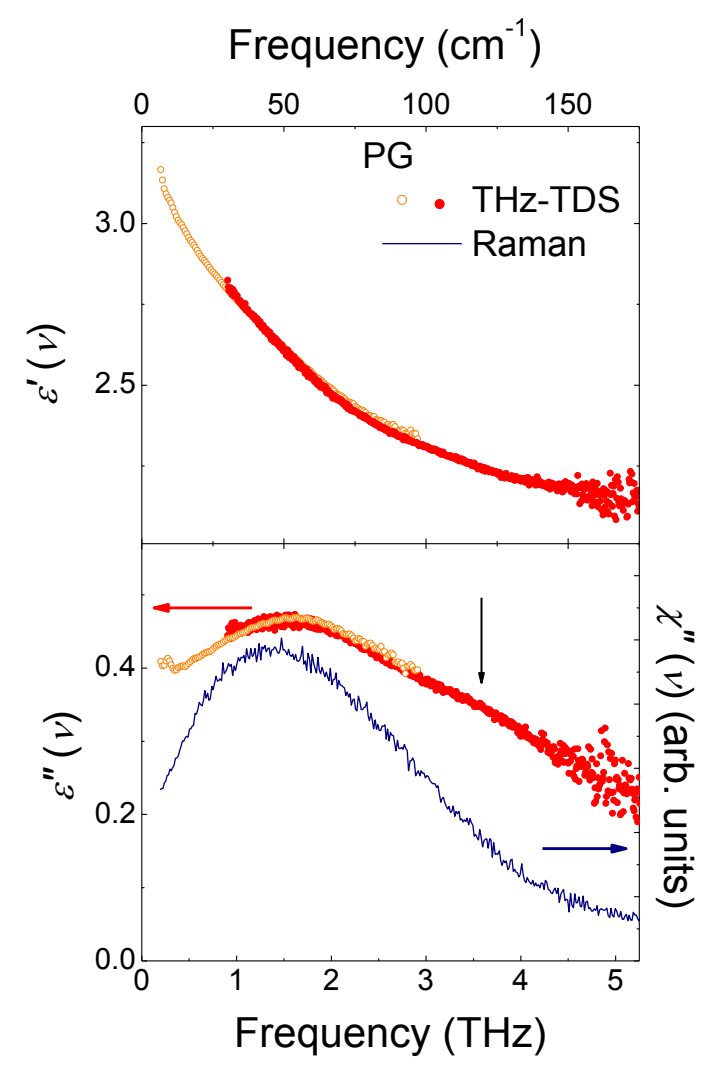

Figure 4. (a) The $\varepsilon$ ' and (b) the comparison between the $\varepsilon$ " and the $\chi$ " (blue solid line) of PG at room temperature. In the complex dielectric constants spectra, the orange circles show the result from RT-10000 and the red dots show the result from TAS7500SU. 


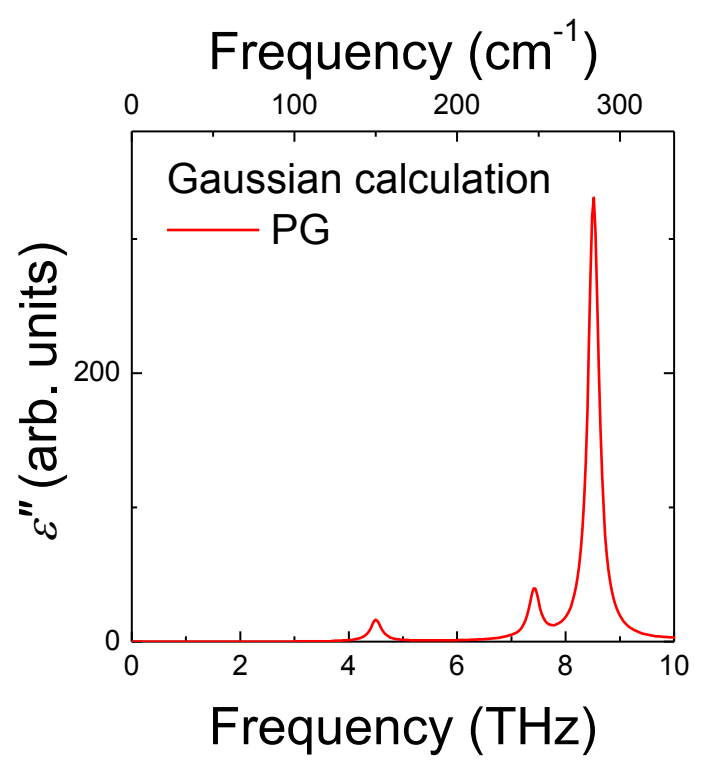

Figure 5. The Gaussian calculation result of PG monomer. The result indicates there is no intra-molecular vibration below $4.5 \mathrm{THz}$.

\section{Conclusion}

We have investigated the broadband terahertz dynamics ( 0.1 to $5.0 \mathrm{THz})$ of the liquid state of PG by using two different THz-TDS systems and Raman scattering equipment. Two THz-TDS systems enable to observe the whole spectral structure of the broad peak at $1.5 \mathrm{THz}$ in PG. The peak at 1.5 $\mathrm{THz}$ is observed both in $\varepsilon$ " and $\chi$ ", while the small shoulder peak at $3.6 \mathrm{THz}$ is only observed in the $\varepsilon$ " spectrum. In contrast to the high frequency region, the low frequency side of the $\varepsilon$ " spectrum of PG includes the contribution from the $\mathrm{GHz}$ region, and the precedent report of the $\mathrm{GHz}$ dielectric measurement supports this suggestion [8]. From the comparison between $\varepsilon$ " and $\chi$ ", the line-shape of the spectra is clearly different, and it suggested that it relates to the motion of the $\mathrm{OH}$ groups which cause the hydrogen bond mediated dipole-dipole interactions.

\section{Acknowledgement}

The authors are very thankful for the technical assistance by H. Takayama, and for the technical support of the broadband THz-TDS measurements by the ADVANTEST Corporation. This study was partially supported by a Grant-in-Aid for Young Scientists (B) (24740194) from JSPS, the Sasakawa Scientific Research Grant from The Japan Science Society, and Casio Science Promotion Foundation.

\section{References}

[1] T. Mori, H. Igawa, D. Okada, Y. Yamamoto, K. Iwamoto, N. Toyota, S. Kojima, J. Mol. Struct. 1090 (2015) 93.

[2] T. Shibata, T. Mori, S. Kojima, Spectrochim. Acta, Part A 150 (2015) 207.

[3] H. Igawa, T. Mori, S. Kojima, Jpn. J. Appl. Phys. 53 (2014) 05FE01.

[4] Y. Yomogida, Y. Sato, R. Nozaki, T. Mishina, J. Nakahara, Physica B 405 (2010) 2208.

[5] N.Y. Tan, R. Li, P. Braeuer, C. D'Agostino, L.F. Gladden, J.A. Zeitler, Phys. Chem. Chem. Phys. 17 (2015) 5999.

[6] T. Shibuya, K. Suizu, K. Kawase, Appl. Phys. Express 3 (2010) 082201.

[7] T. Fukasawa, T. Sato, J. Watanabe, Y. Hama, W. Kunz, R. Buchner, Phys. Rev. Lett. 95 (2005) 197802. 
[8] M. Köehler, P. Lunkenheimer, Y. Goncharov, R. Wehn, A. Loidl, J. Non-Cryst. Solids 356 (2010) 529.

[9] K. Elamin, J. Bjorklund, F. Nyhlen, M. Yttergren, L. Martensson, J. Swenson, J. Chem. Phys. 141 (2014) 034505.

[10]P. Chakravarty, S. Kothari, A. Deese, J.W. Lubach, Mol. Pharm. 12 (2015) 2551.

[11]S. Kojima, T. Mori, T. Shibata, Y. Kobayashi, Pharm. Anal. Acta 6 (2015) 1000401.

[12]E.V. Loewenst, D.R. Smith, R.L. Morgan, Appl. Opt. 12 (1973) 398.

[13]B.D. Saksena, Proc. Ind. Acad. Sci. 12 (1940) 93.

[14]S. Wietzke, C. Jansen, M. Reuter, T. Jung, J. Hehl, D. Kraft, S. Chatterjee, A. Greiner, M. Koch, Appl. Phys. Lett. 97 (2010) 022901.

[15]M. Tasumi, S. Krimm, J. Chem. Phys. 46 (1967) 755.

[16]M.J. Frisch, G.W. Trucks, H.B. Schlegel, G.E. Scuseria, M.A. Robb, J.R. Cheeseman, G. Scalmani, V. Barone, B. Mennucci, G.A. Petersson, H. Nakatsuji, M. Caricato, X. Li, H.P. Hratchian, A.F. Izmaylov, J. Bloino, G. Zheng, J.L. Sonnenberg, M. Hada, M. Ehara, K. Toyota, R. Fukuda, J. Hasegawa, M. Ishida, T. Nakajima, Y. Honda, O. Kitao, H. Nakai, T. Vreven, J.A. Montgomery Jr., J.E. Peralta, F. Ogliaro, M. Bearpark, J.J. Heyd, E. Brothers, K.N. Kudin, V.N. Staroverov, R. Kobayashi, J. Normand, K. Raghavachari, A. Rendell, J.C. Burant, S.S. Iyengar, J. Tomasi, M. Cossi, N. Rega, J.M. Millam, M. Klene, J.E. Knox, J.B. Cross, V. Bakken, C. Adamo, J. Jaramillo, R. Gomperts, R.E. Stratmann, O. Yazyev, A.J. Austin, R. Cammi, C. Pomelli, J.W. Ochterski, R.L. Martin, K. Morokuma, V.G. Zakrzewski, G.A. Voth, P. Salvador, J.J. Dannenberg, S. Dapprich, A.D. Daniels, Ö. Farkas, J.B. Foresman, J.V. Ortiz, J. Cioslowski, D.J. Fox, Gaussian 09, Revision D. 01, Gaussian, Inc. , Wallingford CT, 2009.

[17]W. Hehre, R. Ditchfie, J. Pople, J. Chem. Phys. 56 (1972) 2257.

[18]Y. Yomogida, Y. Sato, K. Yamakawa, R. Nozaki, T. Mishina, J. Nakahara, J. Mol. Struct. 970 (2010) 171.

[19]Y. Yomogida, Y. Sato, R. Nozaki, T. Mishina, J. Nakahara, Physica B 405 (2010) 2208.

[20]T. Perova, D. Christensen, U. Rasmussen, J. Vij, O. Nielsen, Vib. Spectrosc. 18 (1998) 149.

[21]T. Uchino, T. Yoko, Science 273 (1996) 480.

[22]S. Kojima, H. Sato, A. Yoshihara, J. Phys. : Condens. Matter 9 (1997) 10079. 